\title{
Sulforaphane: Mechanisms of Epigenetic Reversion and its Anti-Carcinogenic Pathways as Potential Adjunctive Treatment in Cancer
}

\author{
Andre da Silva Santos ${ }^{1,2}$, Sandra Maria Matta ${ }^{3,4 *}$ and Henry Okigami ${ }^{5}$ \\ ${ }^{1}$ Medical Director, Clinical Oncologist at the Center for Integrative Oncology and \\ Prevention (COIP), São Paulo, Brazil \\ ${ }^{2}$ Scientific Director of G8 Integrative, São Paulo, Brazil \\ ${ }^{3}$ Nutrigeneticist and Registered Dietitian, Clinical Oncologist at the Center for Integrative \\ Oncology and Prevention (COIP), São Paulo, Brazil \\ ${ }^{4}$ Scientific Director in Nutrition/Nutritional Genomics/Oncogenetic of G8 Integrative, São \\ Paulo, Brazil \\ ${ }^{5}$ Director of Science and Technology, Pharmaceutical Biochemist at the Center for \\ Integrative Oncology and Prevention (COIP), São Paulo, Brazil \\ *Corresponding Author: Sandra Maria Matta, Nutrigeneticist and Registered Dietitian, \\ Clinical Oncologist at the Center for Integrative Oncology and Prevention (COIP), São \\ Paulo, Brazil
}

Received: February 29, 2020

Published: May 29, 2020

(C) All rights are reserved by Andre da Silva Santos., et al.

\begin{abstract}
Estimates of cancer in Brazil and the salty world each year, placing it at the top of the death causes ranking.

The first and last year of 2007 was 7.6 million in 2007, showing a 32\% increase in deaths between 2000 and 2007 . In 2007 , the cancer was responsible for $13 \%$ of all deaths in the world [1,2].

Like what is in Brazil, what is a more common cause of death, the elimination of accidents and cerebrovascular disease. In 2014, 395,000 new cases of cancer were found, 205,000 in men and 190,000 in women. The most incident types of cancer are prostate, lung, colon and rectum. In women, the most common cancers are breast, colon and rectum, cervix, lung and thyroid, according to INCA data. Estimate 2014: Incidence of Cancer in Brazil. 2014; Rio de Janeiro: INCA, 124p.

This is a challenge to prevent the preventive decision of the prevention of the risk of the treatment of treatment and treatment of debilitating disease.

Thus, sulforaphane (SFN) is a natural isothiocyanate derived from cruciferous vegetables, such as broccoli, cabbage, watercress, arugula, kale, etc.

This phytoactive product (isothiocyanate) acts in cellular destiny through the nutritionist dietitian, although after an observation that dietetics are not detected in the urine is an indication that they are not absorbed [3]. In addition, there are sessions available so that the apparatus cannot convert glycosinolates to isothiocyanates [4]. However, they include literacy shows that glycosinolates dietetic they best converted in isothiocyanates in animals and humans, and that this migration is mediated by the activity of myrosinase of the enteric microflora $[3,5,6]$. Once generated, isothiocyanates are absorbed and metabolized by sequential enzyme waves, a first of which is a conjugation with glutathione $[7,8]$.
\end{abstract}

Keywords: Sulforaphane (SFN); Isothiocyanate; Cancer

\section{Introduction}

Sulforaphane (SFN) is a natural isothiocyanate derived from cruciferous vegetables, such as broccoli, cabbage, watercress, rocket, kale, etc.

This phytoactive product (isothiocyanate) acts on a cellular target through a nutrigenomic mechanism, but after the observation that dietary glycosinolates are not detected in the urine, it is suggested that they are not absorbed [3]. In addition, available evidence suggests that mammalian tissues cannot convert glycosinolates to isothiocyanates [4]. However, literary evidence shows that dietary glycosinolates are indeed converted into isothiocyanates in animals and humans, and that this conversion is mediated by the myrosinase activity of the enteric microflora $[3,5,6]$. Once generated, isothiocyanates are absorbed and metabolized by sequential enzymatic reactions, the first of which is conjugation with glutathione $[7,8]$.

Because of its efficacy and safety, bioactive SFN is recognized as a promising chemopreventive agent with antitumor effects in cancers, cervical [9], breast [10] and bladder [11]; renal cell carcinoma (RCC) [12]; non-small cell lung cancer (NSCLC) [13] and cancer of the colon and prostate [14]. SFN was reported in a study to increase efficacy at low dose cisplatin (CDDP) [15].

Nutrigenomic mechanisms of anticancer activities of SFN can modulate the tumor cell cycle, through genomic signaling they act in the process of apoptosis and angiogenesis. 
In the cell cycle SFN disrupts the G2/M phase, consequently inhibits the proliferation and growth of the tumor cell. These pathways undergo downregulation of the cyclin B1 [9] and cyclin D1 [16] genes, as well as increased levels of p21WAF1/CIP1 protein [16].

SFN also acts in other ways by increasing the expression of the proapoptotic Bax protein and decreasing the expression of the Bcl$\mathrm{x}$ antiapoptotic protein, thereby inducing apoptosis of cancer cells [17].

This was a major effort because it was recognized that the induction of phase 2 enzymes, such as quinone oxireductase 1 (NQO1) and glutathione S-transferase (GST), is an important strategy to obtain protection against carcinogenesis $[18,19]$.

One of the first tumor-triggering mechanisms is linked to the hypoxia- $1 \alpha$ inducing factor (HIF-1 $\alpha$ ), in studies of the efficacy of a drug known as organometallic ruthenium II, shows the benefit and importance of blocking this pathway in cancer treatment [20].

In addition to HIF- $1 \alpha$, other pathways of suppression of vascular endothelial growth factor 2 (VEGF 2) expression and activity are also attributed to SFN and this pathway evaluated in the study inhibited angiogenesis and metastasis of ovarian cancers and colon $[21,22]$.

Oxidoreduction and epigenetic pathways in cancer

In recent decades, there has been great interest in the role of free radicals, more commonly known as reactive oxygen species (ROS) and reactive nitrogen species (RNS), not only in experimental medicine but also in clinical practice. ROS and RNS: they are generated by several causes, among them, during irradiation by UV light, X-rays and gamma rays, in addition they are reaction products catalysed by metals and are present as pollutants in the atmosphere.

ROS are produced by neutrophils and macrophages during inflammation generating byproducts of electron transport reactions catalyzed by mitochondria and other mechanisms [23].

Exposures to these exogenous agents provide epigenetic reactions, known by changes in gene expression that do not affect the DNA sequence. In mammals, such modifications mainly include DNA methylation, histone modifications (acetylation, phosphorylation and methylation) and regulation of non-coding RNA. Epigenetic changes are reversible and may readily respond to natural bioactive dietary compounds [24], such as SFN, since it has shown regular activation of the gene or the silencing involved in cancer through epigenetic modifications [25].

Epigenetic reactions occur through environmental factors, food, water and air. To reduce the burden of these exposures you need to eliminate them. However, in many cases, exposures are unavoidable, such as exposures to aflatoxins and other mycotoxins in food. With this, it is necessary to have substantial behavioral changes in our living habits or economic investments in the prevention of ex- posures that are extremely difficult to implement in individuals or populations. In such cases, effective, tolerable, low-cost approaches and practices for chemoprevention with glycosinolates-rich foods as precursors of isothiocyanates, glucoraphanin and its isothiocyanic sulforaphane found in broccoli may be especially desirable as anticarcinogenic agents [26].

In the long run, excessive exposure to oxidative stress is one of the main factors promoting carcinogenesis through DNA damage, providing nucleotide pleomorphisms, mutations and chronic cellular inflammation [27].

The activation of Nrf2 by SFN induced the expression of several cytoprotective genes with anticarcinogenic activities [28,29]. Its potential as a strong activator of factor 2 related to erythroid nuclear factor-2 (NF-E2-) 2 (Nrf2) is well known, since Nrf2 is a critical transcription factor in response to oxidative stress [30].

NRf2-mediated genes with cytoprotective activities include antioxidant genes and phase II enzymes, such as nicotinamide adenine dinucleotide (NAD (P) H): quinone oxidoreductase 1 (NQO1), heme oxygenase 1 (HO-1), catalase, glutamate-cysteine ligase (GCL), glutathione $\mathrm{S}$ transferase (GST), UDP-glucuronosyltransferases (UGT) [31], epoxide hydrolase [32,33], ferritin [33] and superoxide dismutase (SOD). Several studies have shown that the effects of SFN on Nrf2 and its downstream cytoprotective genes are through the modification of the cysteine residues Keap1 [34]. Intracellular ROS generation, dose-dependent and time-dependent Bax/Bcl2 change and the high expression proteins of cytochrome C, Casp-3, Casp-8 and PARP-1 demonstrated the apoptotic pathway induced by SFN, as well as induction of apoptosis by phosphorylation of mitogenactivated protein kinases (MAPKs) such as JNK and P-38 [35], phosphatidylinositol 3-kinase (PI3K) and proteinase kinase C (PKC); and epigenetic modifications, which resulted in phosphorylation, nuclear accumulation and increased transcription and stability of Nrf2 [36-39].

Our future prospects through clinical studies in humans have evidences on the chemopreventive effects of SFN on carcinogenesis.

It is noteworthy that several clinical trials evaluated the safety and tolerance of SFN at the doses used. Two phase I clinical trials showed that extracts of broccoli sprouts containing SFN were well tolerated and did not cause significant adverse effects when given orally by healthy volunteers at $25 \mu \mathrm{mol}$ for 7 days or women with breast cancer receiving $200 \mu \mathrm{mol}$ of mean dose 50 min before surgery $[40,41]$.

A recent Phase II clinical study in men with recurrent prostate cancer also confirms the safety of NSF [42].

In another study evaluating the clinical efficacy of SFN in patients with advanced pancreatic ductal adenocarcinoma, data indicated that $90 \mathrm{mg} /$ day of active SFN effectively inhibited tumor growth and increased the sensitivity of cancer cells to chemotherapy [43]. 
In humans, the consumption of SFN-rich broccoli shoots showed a chemopreventive mechanism by inhibiting histone deacetylases (HDACs) in peripheral blood mononuclear cells, which in turn causes an increase in histone acetylation in the promoters of silenced tumor suppressor genes by the epigenetic effect, reactivating the tumor suppressor gene and inducing cell cycle arrest and/or apoptosis $[44,45]$. These clinical studies suggest that SFN is a promising anticancer agent through its potential epigenetic mechanisms.

\section{Conclusion}

Based on the studies described above, it is evident that dietary compound SFN has little or no adverse side effects, in addition to exerting anticarcinogenic activities through multiple mechanisms, including epigenetic regulation. Thus, daily consumption of CFNrich cruciferous vegetables is not only a healthy diet choice, but also an important effective chemopreventive strategy. SFN, as an inducer of Nrf2, shows the ability to re-activate the expression of Nrf2 and its cytoprotective genes as a target in the prevention of carcinogenesis by epigenetic mechanisms.

However, these studies have led us to propose the epigenetic restoration of Nrf2 by SFN as an important strategy against diseases related to oxidative damage, including cancer, in addition to providing new directions of research and preventive approaches to diseases related to oxidative damage.

Therefore, SFN significantly increases cytoprotection through genes involved in various cellular defense mechanisms against cellular toxicities and carcinogenic inductions.

In this review, we present the anticancer activities of SFN and its epigenetic mechanisms, including the epigenetic reactivation of Nrf2. This information will help facilitate the discovery and development of new antineoplastic drugs.

\section{Bibliography}

1. Yllmaz HH., et al. "Cancer trends and incidence and mortality patterns in Turkey". Japanese Journal of Clinical Oncology 41.1 (2011): 10-16.

2. Bray F., et al. "Global estimates of cancer prevalence for 27 sites in the adult population in 2008". International Journal of Cancer 132.5 (2013): 1133-1145.

3. Shapiro TA., et al. "Human metabolism and excretion of cancer chemoprotective glucosinolates and isothiocyanates of cruciferous vegetables". Cancer Epidemiology, Biomarkers and Prevention 7.12 (1998): 1091-100.

4. Fahey JW., et al. "The chemical diversity and distribution of glucosinolates and isothiocyanates among plants". Phytochemistry 56.1 (2001): 5-51.

5. Rabot S., et al. "Rape-seed meal toxicity in gnotobiotic rats: influence of a whole human faecal flora or single human strains of Escherichia coli and Bacteroides vulgatus". British Journal of Nutrition 70.1 (1993): 323-331.
6. Getahun SM and Chung FL. "Conversion of glucosinolates to isothiocyanates in humans after ingestion of cooked watercress". Cancer Epidemiology, Biomarkers and Prevention 8.5 (1999): 447-451.

7. Zhang Y., et al. "Reversible conjugation of isothiocyanates with glutathione catalyzed by human glutathione transferases". Biochemical and Biophysical Research Communications 206.2 (1995): 748-755.

8. Kolm RH., et al. "Isothiocyanates as substrates for human glutathione transferases: structure-activity studies”. Biochemical Journal 311.2 (1995): 453-459.

9. Cheng Y-M., et al. "Sulforaphane, a Dietary Isothiocyanate, Induces $\mathrm{G}_{2} / \mathrm{M}$ Arrest in Cervical Cancer Cells through CyclinB1 Downregulation and GADD45 $\beta / C D C 2$ Association". International Journal of Molecular Sciences 17.9 (2016): 1530.

10. Peng X., et al. "Sulforaphane inhibits invasion by phosphorylating ERK1/2 to regulate E-cadherin and CD44v6 in human prostate cancer DU145 cells". Oncology Reports 34.3 (2015): 1565-1572.

11. Leone A., et al. "Sulforaphane for the chemoprevention of bladder cancer: molecular mechanism targeted approach". Oncotarget 8.21 (2017): 35412-35424.

12. Juengel E., et al. "Sulforaphane inhibits proliferation and invasive activity of everolimus-resistant kidney cancer cells In vitro". Oncotarget 7.51 (2016): 85208-85219.

13. Wang D-X., et al. "Sulforaphane suppresses EMT and metastasis in human lung cancer through miR-616-5p-mediated GSK3 $\beta / \beta$-catenin signaling pathways". Acta Pharmaceutica Sinica 38.2 (2017): 241-251.

14. Clarke JD., et al. "Multi-targeted prevention of cancer by sulforaphane". Cancer Letters 269.2 (2008): 291-304.

15. Wang X., et al. "Sulforaphane improves chemotherapy efficacy by targeting cancer stem cell-like properties via the miR-124/ IL-6R/STAT3 axis". Scientific Reports 6 (2016): 36796.

16. Żuryń A., et al. "The effect of sulforaphane on the cell cycle, apoptosis and expression of cyclin D1 and p21 in the A549 non-small cell lung cancer cell line". International Journal of Oncology 48.6 (2016): 2521-2533.

17. Kim S-H., et al. "Sulforaphane sensitizes human breast cancer cells to paclitaxel-induced apoptosis by downregulating the NF-кB signaling pathway". Oncology Letters 13.6 (2017): 44274432.

18. Talalay P. "Chemoprotection against cancer by induction of phase 2 enzymes". Bio Factors 12.1-4 (2000): 5-11.

19. Talalay P. "Mechanisms of induction of enzymes that protect against chemical carcinogenesis". Advances in Enzyme Regulation 28 (1989): 237-250. 
20. Zhao J., et al. "Hypoxia-Targeting Organometallic Ru(II)-Arene Complexes with Enhanced Anticancer Activity in Hypoxic Cancer Cells". Inorganic Chemistry 57.14 (2018): 8396-8403.

21. Pastorek M., et al. "Sulforaphane reduces molecular response to hypoxia in ovarian tumor cells independently of their resistance to chemotherapy". International Journal of Oncology 47.1 (2015): 51-60.

22. Kim DH., et al. "Sulforaphane inhibits hypoxia-induced HIF-1 $\alpha$ and VEGF expression and migration of human colon cancer cells". International Journal of Oncology 47.6 (2015): 22262232.

23. Cadenas E. "Biochemistry of oxygen toxicity". Annual Review of Biochemistry 58 (1989): 79-110.

24. Meeran SM., et al. "Epigenetic targets of bioactive dietary components for cancer prevention and therapy". Clinical Epigenetics 1.3-4 (2010): 101-116.

25. Gerhauser C. "Epigenetic impact of dietary isothiocyanates in cancer chemoprevention". Current Opinion in Clinical Nutrition and Metabolic Care 16.4 (2013): 405-410.

26. Kensler TW., et al. "Keap1-nrf2 signaling: a target for cancer prevention by sulforaphane". Topics in Current Chemistry 329 (2013): 163-177.

27. Narendhirakannan RT and Hannah MAC. "Oxidative stress and skin cancer: an overview". Indian Journal of Clinical Biochemistry 28.2 (2013): 110-115.

28. Johnson GS., et al. "A functional pseudogene, NMRAL2P, is regulated by Nrf2 and serves as a coactivator of NQO1 in sulforaphane-treated colon cancer cells". Molecular Nutrition and Food Research 61.4 (2017).

29. Liu M., et al. "Nrf2 sensitizes prostate cancer cells to radiation via decreasing basal ROS levels". Bio Factors 41.1 (2015): 52-57.

30. Dinkova-Kostova AT., et al. "Direct evidence that sulfhydryl groups of Keap1 are the sensors regulating induction of phase 2 enzymes that protect against carcinogens and oxidants". Proceedings of the National Academy of Sciences of the United States of America 99.18 (2002): 11908-11913.

31. Basten GP., et al. "Sulforaphane and its glutathione conjugate but not sulforaphane nitrile induce UDP-glucuronosyl transferase (UGT1A1) and glutathione transferase (GSTA1) in cultured cells". Carcinogenesis 23.8 (2002): 1399-1404.

32. Thimmulappa RK., et al. "Identification of Nrf2-regulated genes induced by the chemopreventive agent sulforaphane by oligonucleotide microarray". Cancer Research 62.18 (2002): 5196-5203.
33. Hu R., et al. "Gene expression profiles induced by cancer chemopreventive isothiocyanate sulforaphane in the liver of C57BL/6J mice and C57BL/6J/Nrf2 (-/-) mice". Cancer Letters 243.2 (2006): 170-192.

34. Hu C., et al. "Modification of keap1 cysteine residues by sulforaphane". Chemical Research in Toxicology 24.4 (2011): 515521.

35. Mondal A., et al. "Sulforaphene promotes Bax/Bcl2, MAPK-dependent human gastric cancer AGS cells apoptosis and inhibits migration via EGFR, p-ERK1/2 down-regulation". General Physiology and Biophysics 35.1 (2016): 25-34.

36. Magesh S., et al. "Small molecule modulators of Keap1-Nrf2ARE pathway as potential preventive and therapeutic agents". Medicinal Research Reviews 32.4 (2012): 687-726.

37. Qin S and Hou D-X. "Multiple regulations of Keap1/Nrf2 system by dietary phytochemicals". Molecular Nutrition and Food Research 60.8 (2016): 1731-1755.

38. Zhang C., et al. "Sulforaphane enhances Nrf2 expression in prostate cancer TRAMP C1 cells through epigenetic regulation". Biochemical Pharmacology 85.9 (2013): 1398-1404.

39. Su Z-Y., et al. "Requirement and epigenetics reprogramming of Nrf2 in suppression of tumor promoter TPA-induced mouse skin cell transformation by sulforaphane". Cancer Prevention Research 7.3 (2014): 319-329.

40. Shapiro TA., et al. "Safety, tolerance, and metabolism of broccoli sprout glucosinolates and isothiocyanates: a clinical phase I study". Nutrition and Cancer 55.1 (2006): 53-62.

41. Cornblatt BS., et al. "Preclinical and clinical evaluation of sulforaphane for chemoprevention in the breast". Carcinogenesis 28.7 (2007): 1485-1490.

42. Alumkal JJ., et al. "A phase II study of sulforaphane-rich broccoli sprout extracts in men with recurrent prostate cancer". Investigational New Drugs 33.2 (2015): 480-489.

43. Lozanovski VJ., et al. "Pilot study evaluating broccoli sprouts in advanced pancreatic cancer (POUDER trial) - study protocol for a randomized controlled trial". Trials 15 (2014): 204.

44. Clarke JD., et al. "Comparison of isothiocyanate metabolite levels and histone deacetylase activity in human subjects consuming broccoli sprouts or broccoli supplement". Journal of Agricultural and Food Chemistry 59.20 (2011): 10955-10963.

45. Myzak MC., et al. "Sulforaphane retards the growth of human PC-3 xenografts and inhibits HDAC activity in human subjects". Experimental Biology and Medicine 232.2 (2007): 227-234. 
Assets from publication with us

- Prompt Acknowledgement after receiving the article

- Thorough Double blinded peer review

- Rapid Publication

- Issue of Publication Certificate

- High visibility of your Published work

Website: https://www.actascientific.com/

Submit Article: https://www.actascientific.com/submission.php

Email us: editor@actascientific.com

Contact us: +919182824667 